Check for updates

Cite this: RSC Adv., 2019, 9, 39883

\title{
Graphene platelet reinforced copper composites for improved tribological and thermal properties
}

\author{
Mingxia Wu, ${ }^{a}$ Zhe Chen, ${ }^{a}$ Chunjie Huang, ${ }^{\text {ab }}$ Kunlan Huang, ${ }^{a}$ Kyle Jiang ${ }^{c}$ \\ and Jian Liu (D) *a
}

In this work, investigations were conducted to evaluate a type of graphene platelet-reinforced copper (GPL/Cu) composite for enhanced tribological and thermal properties. The pin-on-disc (steel) results show that the wear loss and the friction coefficient of the composites decrease by nearly $80 \%$ and $70 \%$, respectively, in comparison with those of pure $\mathrm{Cu}$. Thermal conductivity of the composites initially improves substantially by approximately $30 \%$ with a slight loading of 0.25 vol\% GPLs and decreases gradually with a higher content of GPLs. Microstructural analysis reveals that the enhancement in the tribological property is attributed to both the self-lubricating property of GPLs and grain refinement while the improvement in the thermal property is closely associated with the uniform dispersion of GPLs.

Received 1st October 2019

Accepted 25th November 2019

DOI: 10.1039/c9ra07962a

rsc.li/rsc-advances

carbide-coated graphite reinforced $\mathrm{Cu}$ composites using a hot

\section{Introduction}

Copper $(\mathrm{Cu})$ is extensively utilized in disc brakes, contact strips, bearings and electronic packaging materials owing to its good wear resistance, high strength and excellent thermal conductivity at both room and high temperatures. ${ }^{1-10}$ Despite the wide application of copper, there is still an increasing demand for improvement in the tribological and thermal performances of $\mathrm{Cu}$ in various industries. For example, high speed trains require brake pads with both excellent wear resistance and heat conduction properties in order to ensure the safe operation of the trains ${ }^{11}$ and the integration and packaging of electronic components demand improved thermal performance to increase the energy density. ${ }^{5}$ It is broadly recognized that the addition of secondary reinforcements with an excellent thermal property into the metal matrix is able to enhance both the tribological and thermal performances of the material and much effort has been devoted to producing self-lubricating $\mathrm{Cu}$ based matrix composites with outstanding heat dissipation. ${ }^{12-14}$ Many reinforcing fillers were selected and introduced in the $\mathrm{Cu}$ matrix. Chen et al. fabricated niobium diselenide $\left(\mathrm{NbSe}_{2}\right)$ and carbon nanotube (CNT) strengthened $\mathrm{Cu}$ composites by a powder metallurgy process and investigated the friction behavior of the materials. ${ }^{15}$ It was found that the wear rate of the composites was reduced by nearly $50 \%$ in comparison with that of pure $\mathrm{Cu}$ and the stress transfer due to the $\mathrm{CNT}$ and $\mathrm{NbSe}_{2}$ was the main strengthening mechanism. Bai et al. produced boron

${ }^{a}$ School of Mechanical Engineering, Sichuan University, Chengdu, 610065, China. E-mail: liujian@scu.edu.cn

${ }^{b}$ Institute of Mechanics, Materials and Civil Engineering, Université Catholique de Louvain, 1348 Louvain-la-Neuve, Belgium

${ }^{c}$ School of Mechanical Engineering, University of Birmingham, Birmingham, B15 2TT, UK pressing process and reported a $180 \%$ and $42 \%$ improvements in thermal conductivity and flexural strength respectively. ${ }^{1}$ The uniform distribution of the graphite and the close interaction between the reinforcement and $\mathrm{Cu}$ matrix were responsible for the enhancement in the thermal and mechanical performance. Yang et al. obtained the $\mathrm{CNT} / \mathrm{Cu}$ composites using as spark plasma sintering (SPS) furnace and studied the correlation between the dispersion of CNT and electrical conductivity of the composites. ${ }^{16}$ Their results indicated that the homogeneous dispersion CNTs would damage the interconnected conductive networks of $\mathrm{Cu}$, leading to a significantly low electrical conductivity while the composites with aggregation of CNTs tend to possess the optimum conductive performance.

Graphene receives tremendous attention extensively due to its excellent mechanical and functional properties. ${ }^{17-21}$ Compared with traditional choices, e.g., CNT and carbon fibre, graphene possesses many attractive features e.g., a extremely high strength, a low shear resistance, a relatively low cost and a comparably high thermal conduction and dissipation performance. ${ }^{3,4}$ So far, few attempts were made to study the effects of graphene platelets (GPLs) on the tribological performance of $\mathrm{Cu}$ composites. Gao et al. investigated the performance of $\mathrm{Cu}$ matrix composites reinforced with homogeneously dispersed GPLs and reported a $65 \%$ decrease in friction coefficient. ${ }^{4}$ The enhancement in the tribological performance was mainly attributed to the self-lubricant property of the GPLs. Mai et al. produced the GPL/Cu composites utilizing an electrodeposition process and found a $35-40 \%$ decrease in the wear rate. ${ }^{8}$ Besides GPLs, the interfacial bonding between GPLs and $\mathrm{Cu}$ was considered an important factor associated with the tribological performance. Chen et al. studies the relation between GPL content and the properties of GPL/Cu composites and obtained 
a $60 \%$ decrease in the friction coefficient and a continuous decrease in thermal diffusivity and electrical conductivity. ${ }^{2}$ The GPLs were found to be beneficial to wear resistance and reduction in friction, but detrimental to the thermal and electrical performance of the materials.

Although much effort has been conducted to fabricate high performance GPL/Cu composites, few work has been able to achieve simultaneous improvements in the wear resistance and heat dissipation. Therefore, in this study research was performed to fabricate $\mathrm{GPL} / \mathrm{Cu}$ composites with improved tribological and thermal properties using SPS. The effects of GPL content on the microstructure and properties were investigated and the correlation between the GPLs and wear and heat dissipation mechanisms were discussed.

\section{Experiments}

\subsection{Starting materials}

Pure $\mathrm{Cu}$ powder $(99.5 \%, 500 \mathrm{~nm})$ and GPLs powder $(6 \mathrm{~nm}$ in thickness, $20 \mu \mathrm{m}$ in diameter) were used as the starting materials. ${ }^{17}$ Powder mixtures with varied content of GPLs were produced by ball milling for 3 hours at $100 \mathrm{rpm}$ with a ball-topowder mass ratio of 2 .

\subsection{Sintering and tribological experiments}

Pure $\mathrm{Cu}$ powder and GPL/Cu powder mixtures were densified by SPS (SPS-1050, SPS Syntex Inc., Japan) processes at $600{ }^{\circ} \mathrm{C}$ for 5 min under vacuum condition. A heating rate of $100{ }^{\circ} \mathrm{C} \mathrm{s}^{-1}$ and a pressure of $35 \mathrm{MPa}$ were used during the sintering process. The processing parameters are selected based upon the previous studies to ensure samples without significant grain growth can be sintered. ${ }^{22,23}$ The sintered sample is with the dimension of $20 \mathrm{~mm}$ in diameter and $10 \mathrm{~mm}$ in thickness and were cut to form samples of $6 \mathrm{~mm}$ in diameter and $9 \mathrm{~mm}$ in thickness. The wear tests of the materials were performed on a tribometer (MVF-1CC, China) following the ASTM (G99-2005) Standard. A pin-on-disc configuration with the pin of $4.8 \mathrm{~mm}$ in diameter as the sintered material and the GCr15 steel disc of $46 \mathrm{~mm}$ in diameter as the counterpart was used and sliding tests were carried out at a normal load of $20 \mathrm{~N}$, a speed of 50 rpm $\min ^{-1}$ and a sliding time of $60 \mathrm{~min}$. The coefficients of the friction were recorded continuously during the wear process.

\subsection{Materials characterizations}

The density of the sintered sample was determined according to the Archimedes principle. Vickers hardness were obtained with a $1 \mathrm{~kg}$ force. Microstructures were observed using an optical microscopy and SEM and phase constituents were detected by XRD. Raman spectroscopy was obtained using a Raman Microscope with a $512 \mathrm{~nm}$ excitation. Wear loss was examined using a precision microbalance and surface roughness was evaluated using a roughmeter (TR200). The thermal conductivity was obtained by a laser flash thermal analyzer following the ASTM Standard (1461-13) and electrical conductivity was assessed using a conductivity meter (sigma 2008B) following the GB standard (YS/T 478-2005).

\section{Results and discussion}

\subsection{Properties of the GPL/Cu composites}

3.1.1 Tribological performances of GPL/Cu composites. Fig. 1 exhibits the friction coefficient of GPL/Cu composites and evidently the pure $\mathrm{Cu}$ shows the largest value and the addition of GPLs into Cu matrix effectively decreases the friction coefficient. The high coefficient of $\mathrm{Cu}$ may be associated with the work hardening effect of the $\mathrm{Cu}$ and the severe adhesion between friction surfaces. Friction coefficient curves descend with the increasing percentage of GPLs and relatively larger fluctuations are noted for the curves of GPL/Cu composites. The decreasing friction coefficient for the GPL/Cu composites is related to the fact that the GPLs on the friction surface can be a solid lubricant to prevent the two friction surfaces from being in direct contact and the fluctuations in the composites are due to the dispersion of GPLs on parts of the surfaces of GPL/Cu composites. The dependence of the average friction coefficient on the percentage of GPLs is complied in Fig. $1 \mathrm{~g}$ and it decreases continuously with the increasing percentage of GPLS and a significantly large reduction from 0.77 to 0.42 is noted when $1.5 \mathrm{vol} \%$ GPLs is added. With the GPLs content increasing to 2 vol\% GPLs, a lowest coefficient of approximately 0.33 , which is one third of that of pure $\mathrm{Cu}$ is achieved. As noted in other composites with an addition of a solid lubricant, ${ }^{\mathbf{2 4 - 2 6}}$ the significant decrease in the friction coefficient is associated with an considerable increase in the area on the friction surface covered by the lubricating film and the coverage area in the presented study is in relation to content and dispersion of the GPLs. Similar to the friction coefficient, the wear loss and roughness of the surfaces of the composites (Fig. 1h) after the friction test exhibit downward trends. Though pure $\mathrm{Cu}$ exhibits a large fluctuating friction coefficient (Fig. 1a), the weight loss during the wear process is not considerably high (Fig. 1h). This can be attributed to the high ductility of the $\mathrm{Cu}$, which can absorb the energy through the plastic deformation and delay the formation of cracks on the friction surfaces or subsurfaces in the wear process while the downward trend indicates that a higher percentage of GPLs tend to cause a lower wear loss and a finer surface for the composites and further corroborates the effectiveness of GPLs in enhancing the wear resistance of the materials.

3.1.2 Mechanical and functional properties of GPL/Cu composites. Fig. 2 exhibits the dependences of density, hardness, and electrical and thermal conductivity (TC) on the percentage of GPLs. As expected, when a secondary reinforcement is added, the densification process is considerably impeded and the relative density of GPL/Cu composites shows a decreasing trend with the continuous increasing percentage of the GPLs. Nevertheless, all of the composites show relative density higher than 95\%, suggesting a well consolidation process is achieved during the SPS. In contrast to the relative density, hardness initially presents a substantial increase with a slight loading of $0.5 \mathrm{vol} \%$ GPLs and continues to increase to the maximum value, which is then followed by a continuous decrease with the further loading of the GPLs. The reason why 

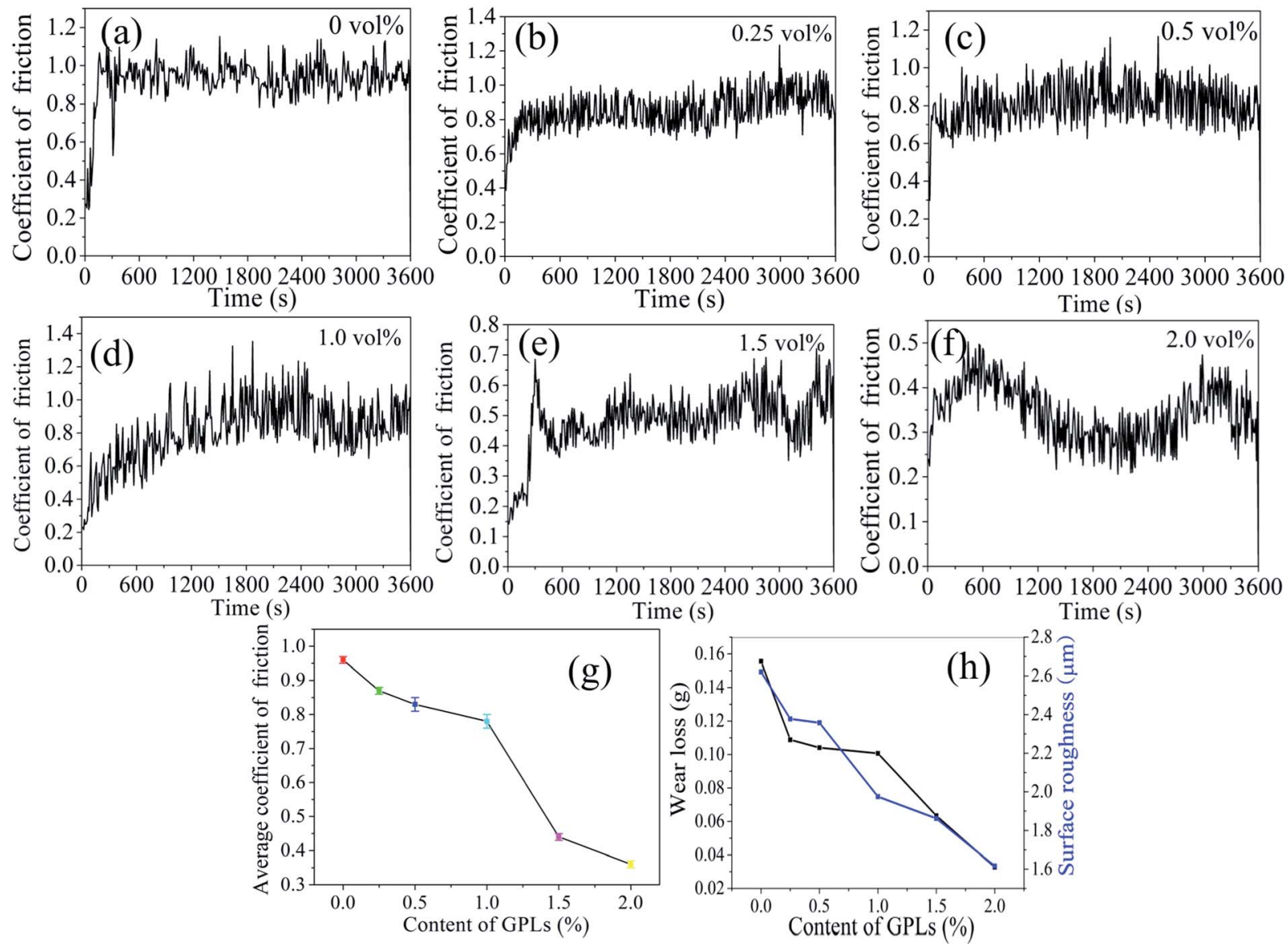

Fig. 1 Variation of friction coefficient $(\mathrm{a}-\mathrm{g})$, wear loss and roughness of surfaces (h) with the content of GPLs. (a) 0 vol\%, (b) 0.25 vol\%, (c) 0.5 vol\%, (d) 1 vol\%, (e) 1.5 vol\%, (f) 2 vol\%.

the hardness exhibits this pattern can be attributed to the degree of densification and reinforcing effects induced by GPLs and a high density and a uniform distribution of GPLs usually tend to result in a high hardness.

Functional properties of the GPL/Cu composites are given in Fig. 2b. It is noted that electrical property decreases continuously when the percentage of GPLs increases from 0 to $1.5 \mathrm{vol} \%$ and a further addition of GPLs to $2 \mathrm{vol} \%$ results in a slight recovery. Clearly the electrical conductivity of all the GPL/Cu composites is lower than that of pure $\mathrm{Cu}$, which suggests that $\mathrm{Cu}$ metal, in light of electrical performance, is negatively affected irrespective of content of the GPLs added. The result agrees well with the previous reports ${ }^{27,28}$ that indicate the introduction of carbon fillers such as CNTs and GPLs would
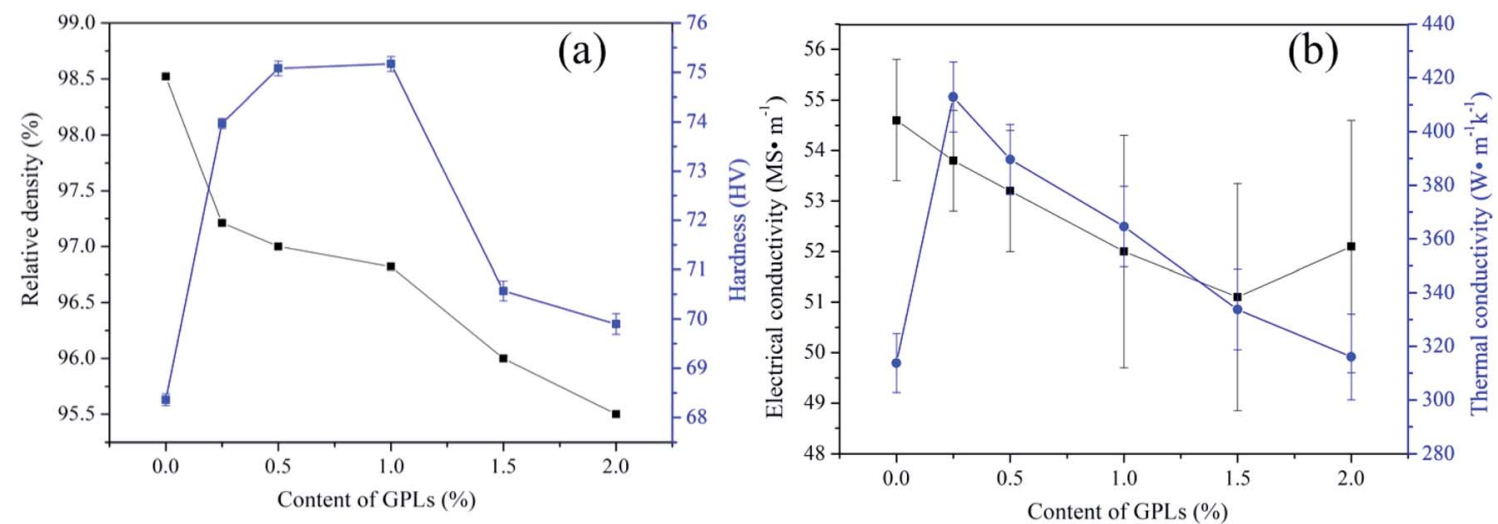

Fig. 2 Variation of mechanical and functional properties with the content of GPLs. (a) Variation of the relative density and hardness with the GPL content. (b) Variation of the electrical and thermal conductivity with the GPL content. 


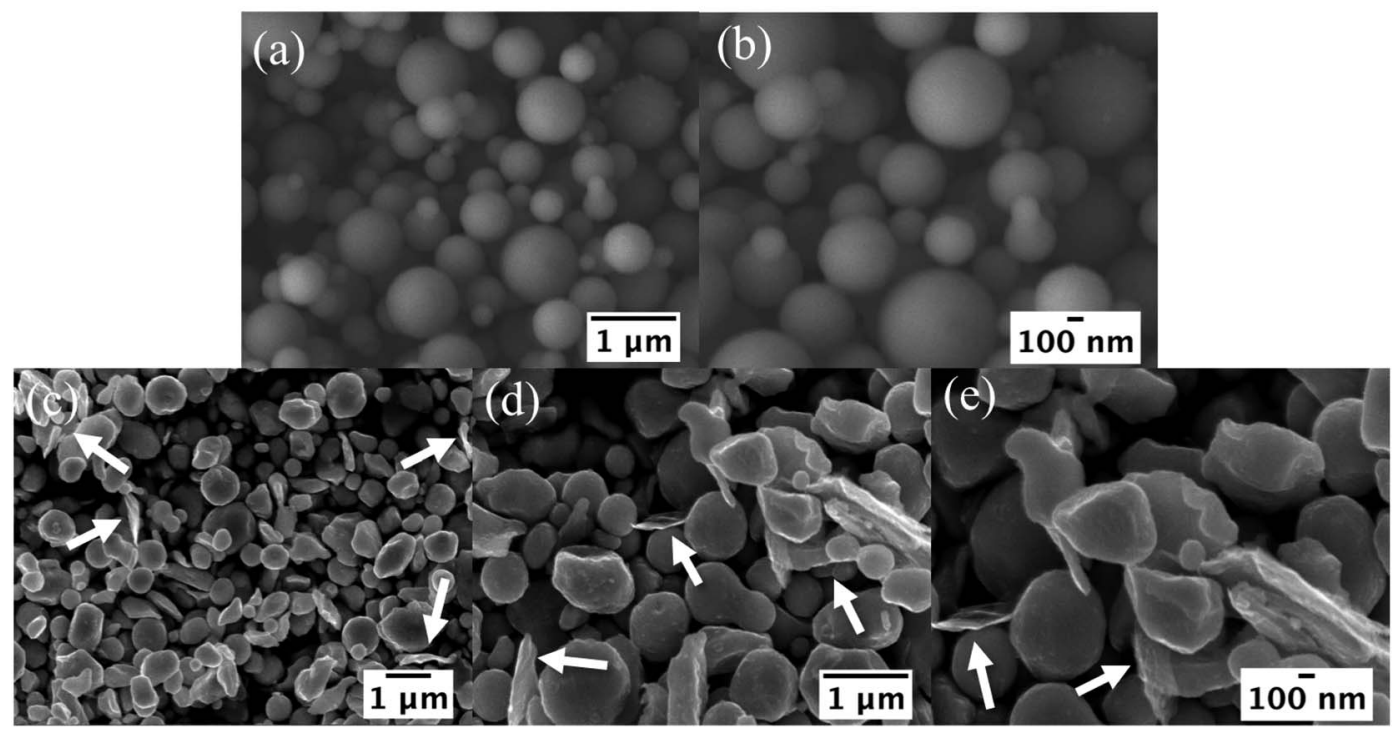

Fig. 3 SEM images of the pure $\mathrm{Cu}(\mathrm{a}$ and $\mathrm{b})$ and GPL/Ti powder mixture ((c-e), white arrows point to GPLs, (e) is a higher magnification image of (c)).

result in the formation of dislocations around the interface between the metal matrix and carbon fillers and increases the possibility of electron scattering. On the contrary to the electrical performance, a minor addition of GPLs leads to a substantial $30 \%$ improvement in the thermal conductivity. Although a further increasing content of GPLs leads to a continuous deterioration in the thermal performance, thermal conductivity of all of the GPL/Cu composites is higher than that of pure $\mathrm{Cu}$, suggesting the addition of GPLs is appreciably effective in improving the thermal performance of the $\mathrm{Cu}$ matrix. The excellent thermal performance of the composites can be attributed to the high thermal conductivity of GPLs and close interfaces between the GPLs and Cu matrix which can facilitate phonon transfer from $\mathrm{Cu}$ to GPLs.

\subsection{Microstructures of the GPL-reinforced $\mathrm{Cu}$ composites}

3.2.1 GPL/Cu powder mixtures. The SEM images of the pure $\mathrm{Cu}$ powder and GPL-Cu powder mixtures are shown in Fig. 3. As noted, the original pure $\mathrm{Cu}$ particles exhibit typical spherical shape with sizes ranging from approximately $100 \mathrm{~nm}$ to $800 \mathrm{~nm}$ while those in the powder mixture appear to be deformed with a smaller size range, which implies deformation

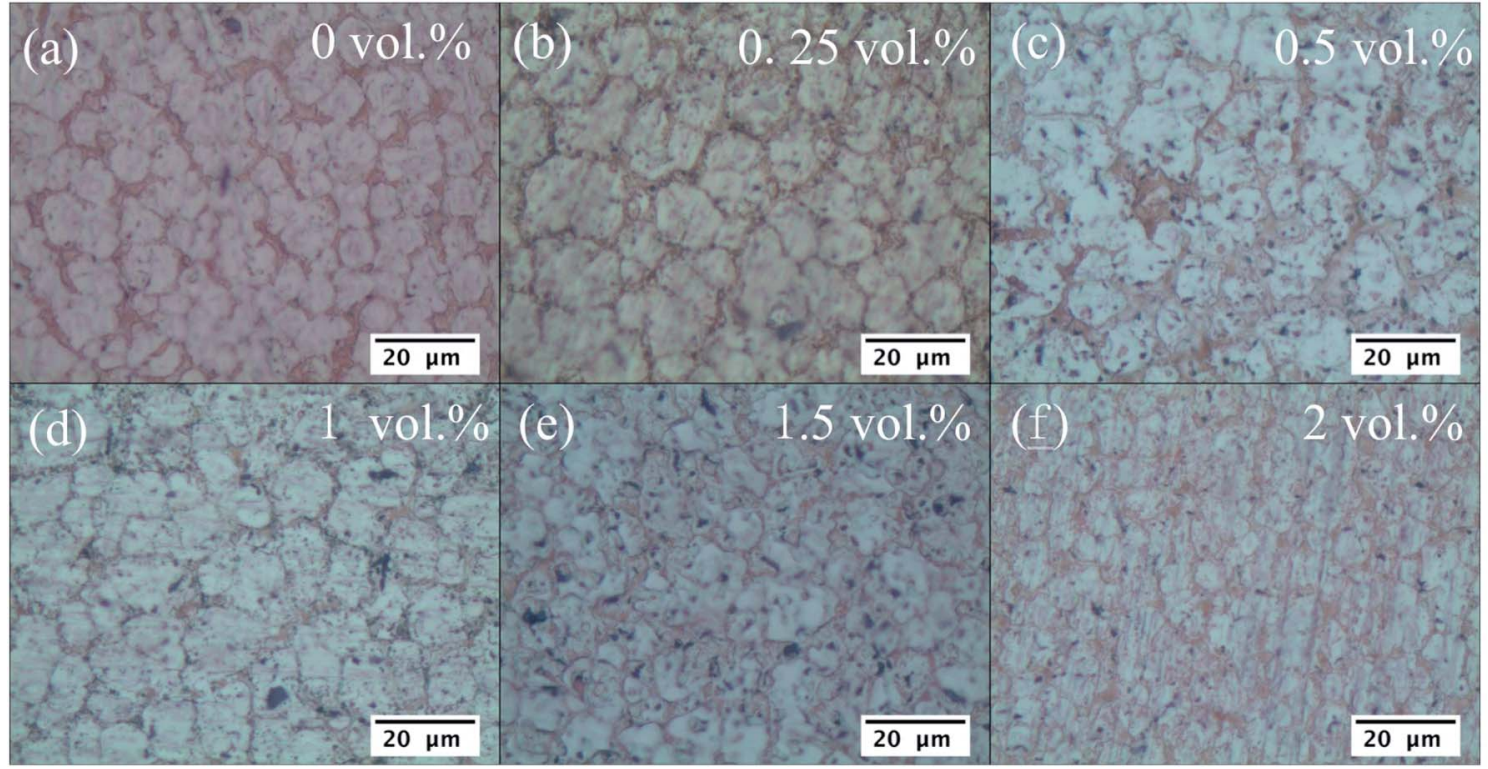

Fig. 4 Optical images of the polished surface of the GPL/Cu composites (black spots indicate the GPLs). (a) 0 vol\%, (b) 0.25 vol\%, (c) 0.5 vol\%, (d) 1 vol\%, (e) 1.5 vol\%, (f) 2 vol\%. 
or even fracture of $\mathrm{Cu}$ particles occurred in the ball milling process. It suggests that the ball milling will cause cold working and cold welding on metal powder ${ }^{29}$ and the cold working will either deform or fracture the powder while the cold welding favors the agglomeration of the particles. Similar to other carbon fillers, GPLs can act as a milling aid distributed between the metal powder and create a network of weak interfaces, ${ }^{30}$ which can be crack initiators to prevent the formation of $\mathrm{Cu}$ and GPL agglomerates, leading to an improved dispersion of the powder mixture.

3.2.2 Surface observations of the sintered GPL-reinforced Cu composites. Optical images of the polished surface of the GPL/Cu composites are given in Fig. 4 and evidently GPLs are uniformly distributed in the $\mathrm{Cu}$ matrix and the composites exhibit finer microstructures with increasing content of GPLs. A further observation of the microstructure is shown in Fig. 5 and a significant reduction in grain size is noted when the volume fraction of GPLs is higher than $1 \%$. It is expected that a fine microstructure induced by GPLs would considerably strengthen the $\mathrm{Cu}$ matrix and contribute to the significant enhancements in mechanical and tribological performances. However, a refined microstructure with a high density of grain boundaries and the dislocations and pores formed during the sintering process would provide many scattering centers for electron transporting, leading to a pronounced decline in the electrical conductivity. It should be noted that contrary to the widely acknowledged perception that a homogenous distribution of carbon fillers would result in the improvements in the properties of the composites. However, the composites with an aggregation rather than a well distribution of GPLs tend to possess a high electrical conductivity. ${ }^{16}$ This phenomenon is attributed to the fact that the conductive path of closely interconnected $\mathrm{Cu}$ is thoroughly damaged by well distributed GPLS by the scattering in the interface between GPLs and Cu. Thus, the electrical conductivity of the composites exhibits a general descending trend with the increasing volume fraction of GPLs. Nevertheless, when a high content of 2 vol\% GPLs is introduced, it may facilitate the formation of aggregates and render the electrical conductivity to bounce back to level higher than that when relatively smaller loadings ( 1 vol\% and $1.5 \mathrm{vol} \%$ ) are introduced. The descent of thermal diffusivity induced by GPLS is associated with the three factors. First, a reduction in the matrix grain size and an increase in the dislocation density leads to a reduction in the mean free path of the heat carrier. ${ }^{31}$ Second, the large thermal expansion mismatch and poor adhesion between the $\mathrm{Cu}$ and carbon filler raise the interfacial thermal resistance. ${ }^{32}$ Third, the formation of pores and voids in the sintering process can serve as insulating barriers and prevent the heat dissipation.

3.2.3 Surfaces of the GPL-reinforced Cu composites after the tribological tests. SEM images of the worn surfaces are studied and shown in Fig. 6. It is evident that a highly rough worn surface with plenty of wear debris is observed for the sintered pure $\mathrm{Cu}$. It is expected that the friction during the tribological tests tends to result in a high temperature on the contact surface and oxidize the worn surfaces. The oxide inclusions formed during the friction process would restrict the movement of the dislocations formed due to the plastic deformation on the interface, which causes the concentration of stress and strain on the sub-surface. ${ }^{33}$ In particular, once the local stress is larger than the rupture strength of the material, cracks and a large wear loss would be produced. With the increasing addition of GPLs, the friction coefficient decreases and a relatively low temperature will be generated on the contact surfaces. As a result, less oxides will be formed, allowing a higher degree of plastic deformation before the rupture of the surfaces. Therefore, a less wear loss would be produced and an introduction of a higher content of GPLs tends to contribute to

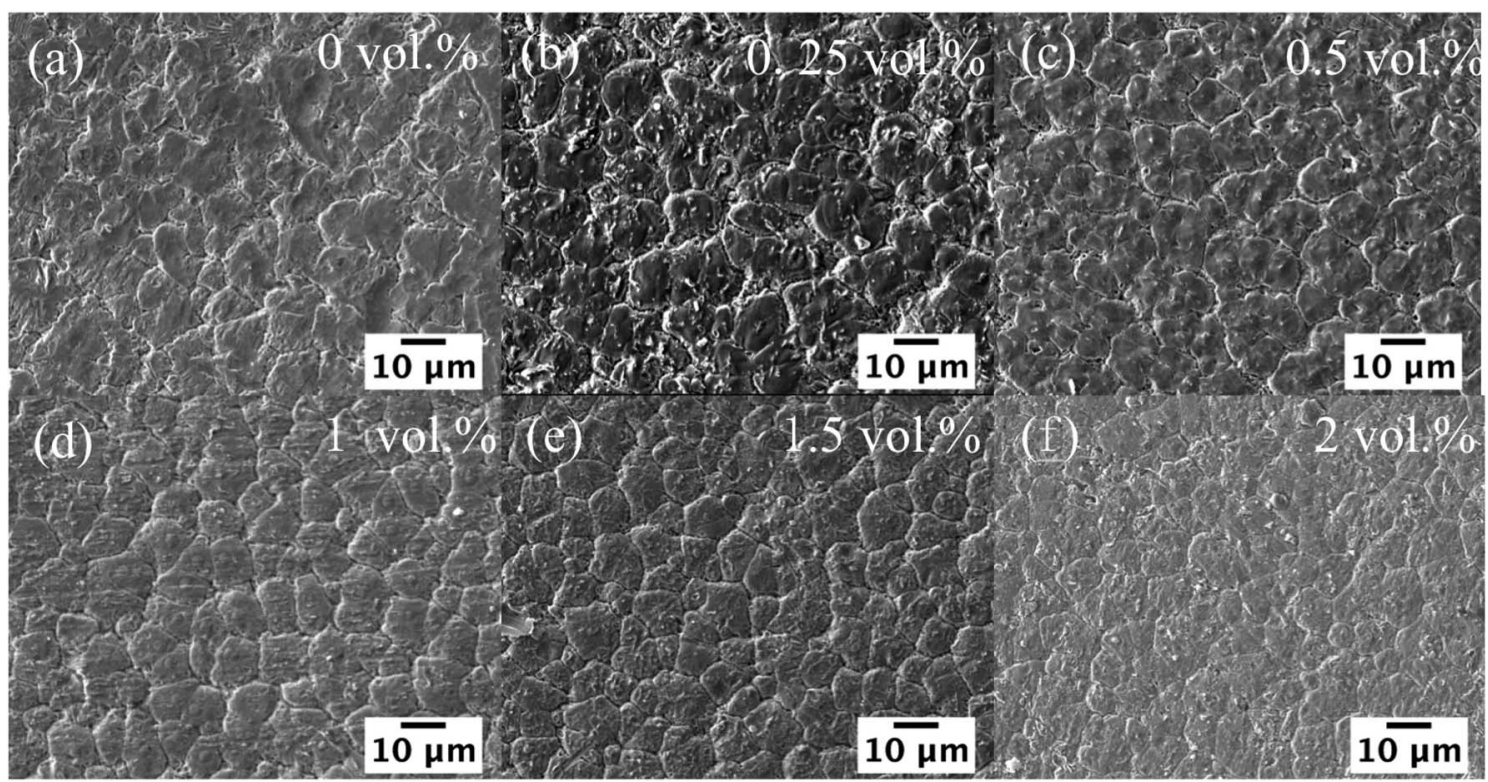

Fig. 5 SEM images of the polished surface of the GPL/Cu composites. (a) 0 vol\%, (b) 0.25 vol\%, (c) 0.5 vol\%, (d) 1 vol\%, (e) 1.5 vol\%, (f) 2 vol\%. 
a smoother surface. A further observation (Fig. $6 \mathrm{~g}$ and $\mathrm{h}$ ) reveals that GPLs may be squeezed out of the composites and distributed on the surfaces. The distribution of the GPLs on the contact surface not just prevents the $\mathrm{Cu}$ from being oxidized but also reduces friction because of the low shear strength of GPLs. When thin GPLs are discontinuous, they are swiftly worn out due to the friction, which explains an unappreciable reduction in the friction coefficient when a low percentage of GPLs is introduced. However, when a high content GPLs is added, GPLs-rich films become continuous and are likely to be formed on the wear track, which would significantly reduce the friction and wear.

3.2.4 XRD patterns and Raman spectroscopy of the GPLreinforced $\mathrm{Cu}$ composites. XRD results of the sintered pure $\mathrm{Cu}$ and GPL/Cu composites are shown in Fig. 7a. Only typical peaks for $\mathrm{Cu}$ are observed and peaks for GPLs and new phases are not detected. The explaination for this observation can be attributed to the low percentage of the carbon filler added that render it difficult trace the reaction products. In addition, shifting of peaks is noted and is associated with the residual stress formed during the cooling process due to different thermal expansion coefficients. Raman spectroscopy of the GPL in the composites are given in Fig. 7b. Three distinctive peaks indicating D, G and
$2 \mathrm{D}$ bands of the graphitic structure are observed and the $\mathrm{G}$ band is considerably higher than the D band. It is suggested that the graphene structure can be assessed based on the ratio between the intensities of D, G and $2 \mathrm{D}$ bands. Specifically $I_{\mathrm{D}} / I_{\mathrm{G}}$ and $I_{2 \mathrm{D}} / I_{\mathrm{G}}$ indicate the defect density and the number of layers in GPLS respectively. ${ }^{19}$ Raman parameters are thus summarized in Table 1 to further analyze the change in graphene structure. It is clear that the values of $I_{2 \mathrm{D}} / I_{\mathrm{G}}$ and $I_{\mathrm{D}} / I_{\mathrm{G}}$ of GPLs decrease with the increasing content of GPLs in the composites, indicating a high volume fraction of GPLs would favor the stacking of graphene sheets and lead to less defects during the ball milling process. Meanwhile, G and 2D bands of GPLs in the composites shift to higher wave number when a higher fraction of GPLs is introduced. The shifting of $\mathrm{G}$ band can be mainly explained by the residual thermal stress on GPLs due to different thermal expansion coefficients.

The dispersion of GPLs is a significantly important factor to ensure the full play of the strengthening effects and functional properties of the GPLs reinforcement. It is observed in Fig. 8a GPLs are uniformly distributed in $\mathrm{Cu}$ (Fig. 8a) and thin GPLs are anchored or dispersed in the grain boundaries of the matrix grains (Fig. 8b-d), which indicates that a good dispersion of GPLs is achieved in the $\mathrm{Cu}$ matrix. The uniform dispersion of

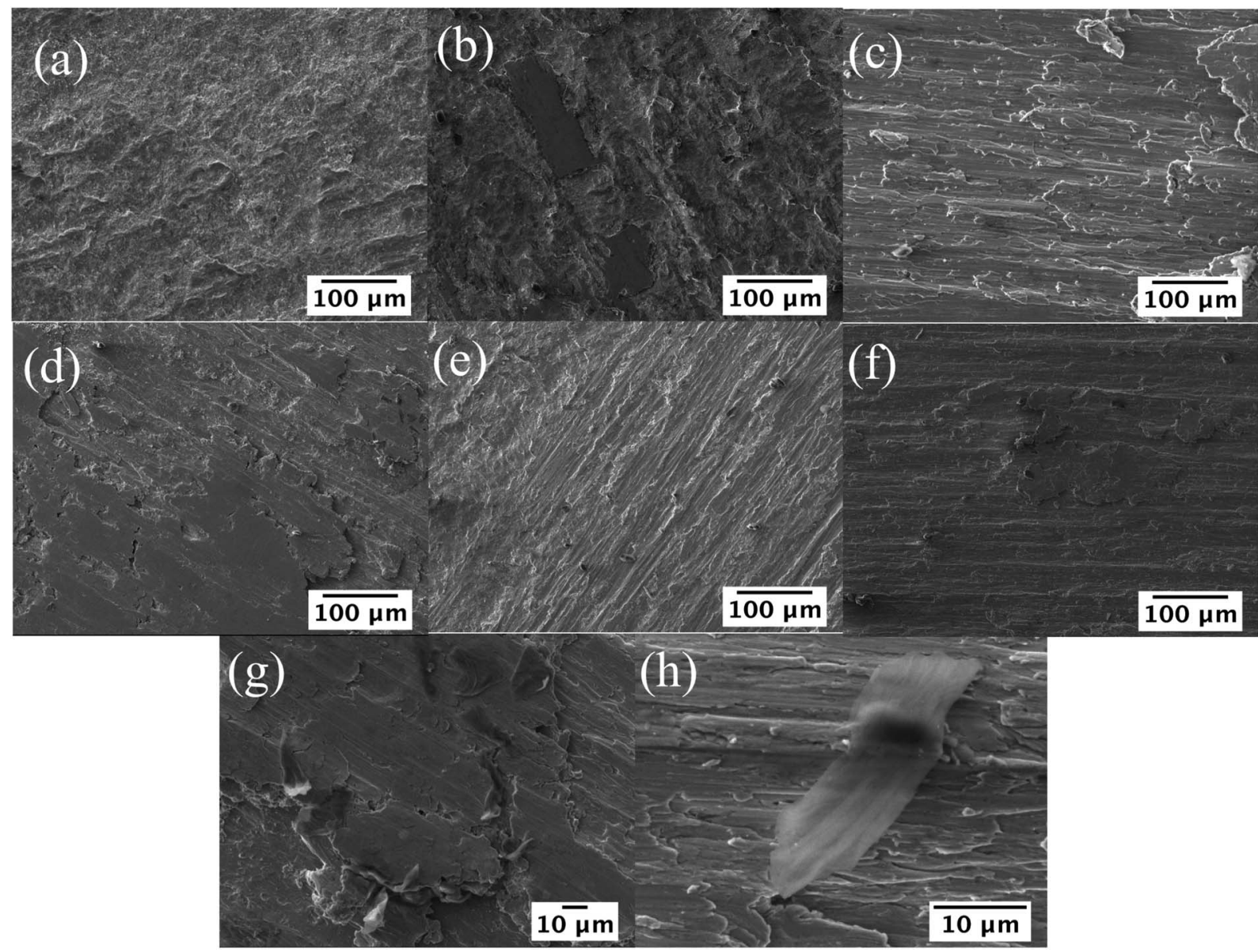

Fig. 6 SEM images of the worn surfaces of the GPL/Cu composites. (a) Pure Cu, (b and g) 0.25 vol\%, (c) 0.5 vol\%, (d) 1 vol\%, (e and h) 1.5 vol\%, (f) 2 vol\%. 

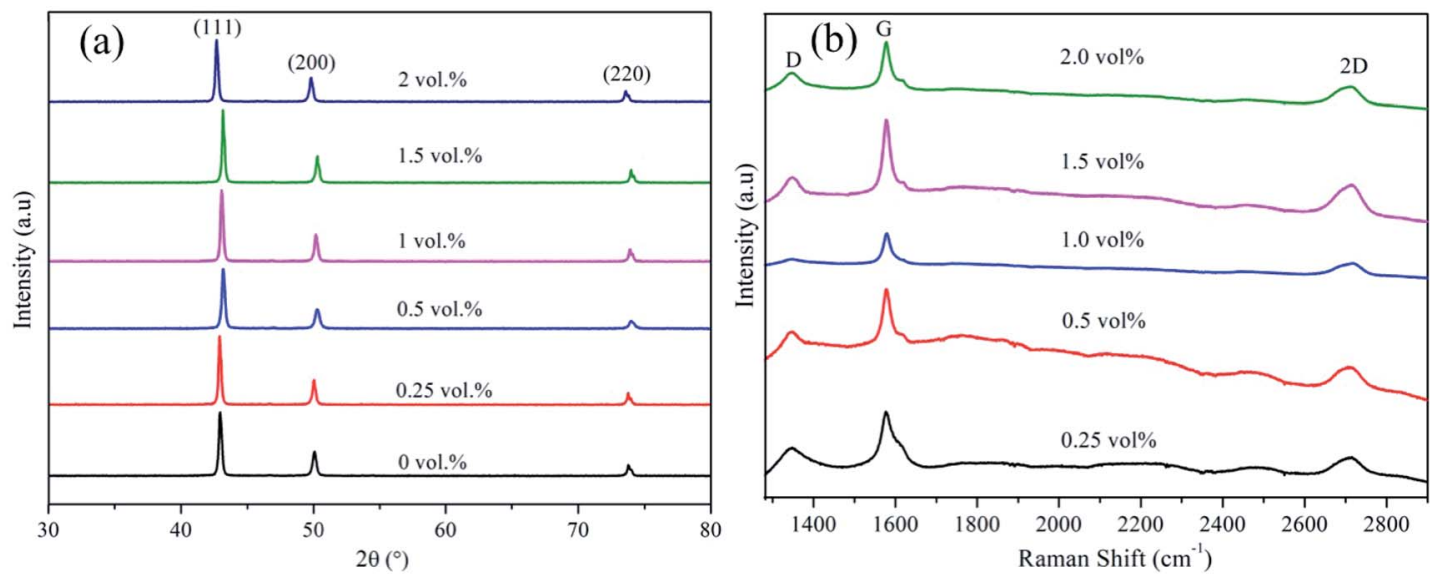

Fig. 7 XRD patterns (a) and Raman spectroscopy (b) of the GPL-reinforced Cu composites.

GPLs tends to result in a refined microstructure and strengthen the $\mathrm{Cu}$ matrix due to the dispersion strengthening mechanism and the high modulus of the GPLs. On the other hand, the introduction of GPLs can alter the wear mechanism of the $\mathrm{Cu}$ matrix. For the reference pure $\mathrm{Cu}$ sample, rupture features with fine debris are noted on the worn surface (Fig. 6a), which indicates severe plastic deformation and oxidation occur during the sliding process. As the friction process continues, the worn surface is oxidized due to the high temperature generated in the contact surface and the oxide inclusions tend to pin the dislocations formed in the vicinity of the interface due to the plastic deformation, which leads to a considerably high stress at local area. Subsequently, surface rupture occurs when the local stress is higher than the rupture strength, resulting in a large wear loss and the formation of a high degree of deep plough and plastic deformation. ${ }^{9}$ The worn appearance of the sintered pure $\mathrm{Cu}$ clearly indicates that adhesive and abrasive wear mechanisms dominate the frictional and wear processes. Nevertheless, a minor addition of the GPLs changes the worn appearance significantly and lubricating layers are formed notably on part of the worn surface (Fig. 6h). These dispersed films can act as lubricants to ease the sliding process and keep the $\mathrm{Cu}$ matrix from adhesion with the steel disc. In addition, the wear depth of the $0.25 \mathrm{vol} \% \mathrm{GPL} / \mathrm{Cu}$ composites is significantly smaller than that of the pure $\mathrm{Cu}$ sample (Fig. 6b), indicating that a slight incorporation of GPLs can considerably improve the wear resistance of the $\mathrm{Cu}$ matrix. A further increasing the content of the GPLs to $0.5 \mathrm{vol} \%$ leads to the formation of parallel grooves on the worn surface in addition to the wear debris produced along the direction of sliding (Fig. 6c). The grooves are expected to be formed due to the micro-ploughing of hard asperity of counter surface or wear debris on the soft $\mathrm{Cu}$, which suggests the abrasive wear rather than the adhesive wear occurs during the most part of the sliding process. When the addition of GPLs reaches to 1 vol\% (Fig. 6d), delamination patterns with large lubricating layers are noted (Fig. $6 \mathrm{~g}$ ) and the delamination wear can be attributed to the combined effects of porosity within the composite, the relatively poor interfacial bonding between $\mathrm{Cu}$ matrix and GPLs and severe plastic deformation. It is expected that cracks formed around the residual pores and the poor interfacial bonding between the $\mathrm{Cu}$ matrix and GPLs reinforcements tend to lead to a boost in delamination wear along with the severe plastic deformation. Further additions of GPLs result in more smooth wear surfaces (Fig. 6e and f) with fine parallel grooves and small wear debris, indicating the abrasive wear mechanism dominates the sliding process. The small wear debris agrees well with the lower wear loss of the GPL/Cu composites (Fig. 1h) and fine grooves on the worn surface. Meanwhile, the number of grooves decreases and the depth of grooves is reduced, which is mainly attributed to the decrease in the number of abrasive wear debris. In particular, the composite with the highest content of the GPLs exhibits the smallest depth of grooves and parts of the surfaces of the composite are covered with some thin lubricating layers which is sharp contrast with those thick delamination layers observed in $1 \mathrm{vol} \% \mathrm{GPL} / \mathrm{Cu}$ composite (Fig. 6d). This fact implies that friction force causes the shear stress in the shallow surface for the 2 vol\% GPL/Cu composite, which is consistent with the lowest friction coefficients in Fig. 1f and lowest wear loss in Fig. 1h. Meanwhile, GPLs tend to be extruded out from the subsurface and smeared on the surface of $\mathrm{Cu}$ matrix (Fig. 6f) during the friction process. ${ }^{34}$ Owing to the lamellar structure and weak van der Waals' force between layers, GPLs can split into many thin sheets under a low shear force during the sliding process and cover a significantly large surface area through the continuous splitting. These thin graphene nanoplatelets are closely attached on the sliding surface and form a continuous GPLs lubricating film, considerably reducing the friction

Table 1 Raman parameters of the GPLs in the GPL/Ti composites

\begin{tabular}{lccccc}
\hline GPLs content & $\nu_{\mathrm{D}}$ & $\nu_{\mathrm{G}}$ & $\nu_{2 \mathrm{D}}$ & $I_{\mathrm{D}} / I_{\mathrm{G}}$ & $I_{2 \mathrm{D}} / I_{\mathrm{G}}$ \\
\hline $0.25 \mathrm{vol} \%$ & 1339 & 1576 & 2712 & 0.77 & 0.57 \\
$0.5 \mathrm{vol} \%$ & 1338 & 1583 & 2715 & 0.70 & 0.58 \\
$1.0 \mathrm{vol} \%$ & 1337 & 1585 & 2717 & 0.63 & 0.58 \\
$1.5 \mathrm{vol} \%$ & 1337 & 1585 & 2729 & 0.63 & 0.64 \\
$2.0 \mathrm{vol} \%$ & 1336 & 1583 & 2731 & 0.60 & 0.72
\end{tabular}


coefficient and leading to the formation of fine features on the worn surface. Overall, the positive effect of the GPLs in improving the wear performance of the composites is because of the formation of a large lubricating film on the wear surface while the adverse influence of the addition of GPLs is associated with the formation of porosity and cracks, resulting in the enhancement in the delamination wear and degrading the mechanical properties. Usually the former plays a leading role in the descending stage of the wear rate while the latter dominates in the ascent stage. The effects of GPLs on the tribological properties can be further corroborated by the observation that layers of black substances are formed on the sliding surface (Fig. 8e-g) of the composites with a relatively lower content of GPLs while they are hardly noted (Fig. 8h-j) when a higher fraction of GPLs is added. It is expected that these black substances are iron oxides (ferrous oxide or ferroferric oxide) and are produced owing to the high temperature generated on the sliding surface as a result of the large friction coefficient whereas a higher content of GPLs leads to a lower temperature on the sliding surface and prevents the formation of these oxides. These oxides and GPLs, as lubricating materials are helpful in reducing the adhesive wear and in particular, a high content of GPLs tends to change the wear mechanisms from the adhesive to the abrasive.

For the thermal conductivity (TC), anisotropy relating to the GPL alignment is present in the GPL/metal composites and usually the in-plane TC $\left(K_{\|}\right)$is much higher than through-plane TC $\left(K_{\perp}\right) .{ }^{13}$ In particular, the TC of the composites can be maximized if an alignment of graphene can be achieved, which makes use of the extraordinary in-plane TC of graphene and minimal interfacial thermal resistance $\left(R_{\mathrm{k}}\right)$. Previous reports indicate $K_{\|}$can be assessed by an effective medium approximation (EMA) model. ${ }^{35}$

$$
K_{\|}=K_{\mathrm{m}}\left\{\frac{2+f_{\mathrm{g}}\left[\beta_{\|}\left(1+\left\langle\cos ^{2} \theta\right\rangle\right)\right]}{2-f_{\mathrm{g}}\left[\beta_{\perp}\left(1-\left\langle\cos ^{2} \theta\right\rangle\right)\right]}\right\}
$$

$$
\beta_{\|}=\frac{K_{\mathrm{g}}}{K_{\mathrm{m}}}-1, \quad \beta_{\perp}=1-\frac{K_{\mathrm{m}}\left(R_{\mathrm{K}} K_{\mathrm{g}} / t+1\right)}{K_{\mathrm{g}}}
$$

where $K_{\mathrm{m}}$ is the TC of the matrix, $K_{\mathrm{g}}$ is the intrinsic in-plane TC of graphene and $t$ is the thickness. $\cos ^{2} \theta$ is the alignment degree. $f_{\mathrm{g}}$ is the percentage of the GPLs.

It is noted from the above equation that both $\left\langle\cos ^{2} q\right\rangle$ and $R_{\mathrm{K}}$ are essential for $K_{\|}$and the $K_{\|}$can be increased by either increasing $\left\langle\cos ^{2} q\right\rangle$ or reducing $R_{\mathrm{K}}$. In addition, the composites with a higher alignment degree can result in a lower in-plane $R_{\mathrm{K}}$. Reportedly the SPS process can induce the preferred selfalignment of GPLs perpendicular to the pressure axis, which helps the attainment of a high TC. ${ }^{13}$ Therefore, it is expected that $R_{\mathrm{K}}$ is the most important influencing parameter for TC. Various factors such as porosity and the dispersion of GPLs are closely associated with the TC. In particular, pores are highly likely to be formed around the interface between the reinforcement and matrix in either GPL/metal or GPL/ceramic composites $^{\mathbf{1 8}}$ due to the poor interfacial wetting ability, which poses a challenge for the fabrication of GPL/Cu composites with high TC. Meanwhile, a well dispersion of GPLs in the matrix is essential for a high TC as it reduce the degree of agglomeration and the likelihood of formation of pores. In the presented study, it is noted that a slight addition of GPLs results in the maximum improvement in TC while further increasing the volume fraction of GPLs leads to continuous deterioration in the thermal performance, which indicates that a high content of GPLs tends to promote the formation of GPL agglomeration and increase the porosity. The associativity among $\mathrm{Cu}$ grains is interrupted with the presence of these pores and therefore GPL/Cu composites exhibit a lower value in TC with a higher percentage of GPLs.

Since the electrical conductivity of graphene is far better than that of $\mathrm{Cu}$, many attempts have been tried to fabricate $\mathrm{GPL} / \mathrm{Cu}$ metal matrix composites to improve the electrical conductivity considerably. However, the results usually turns

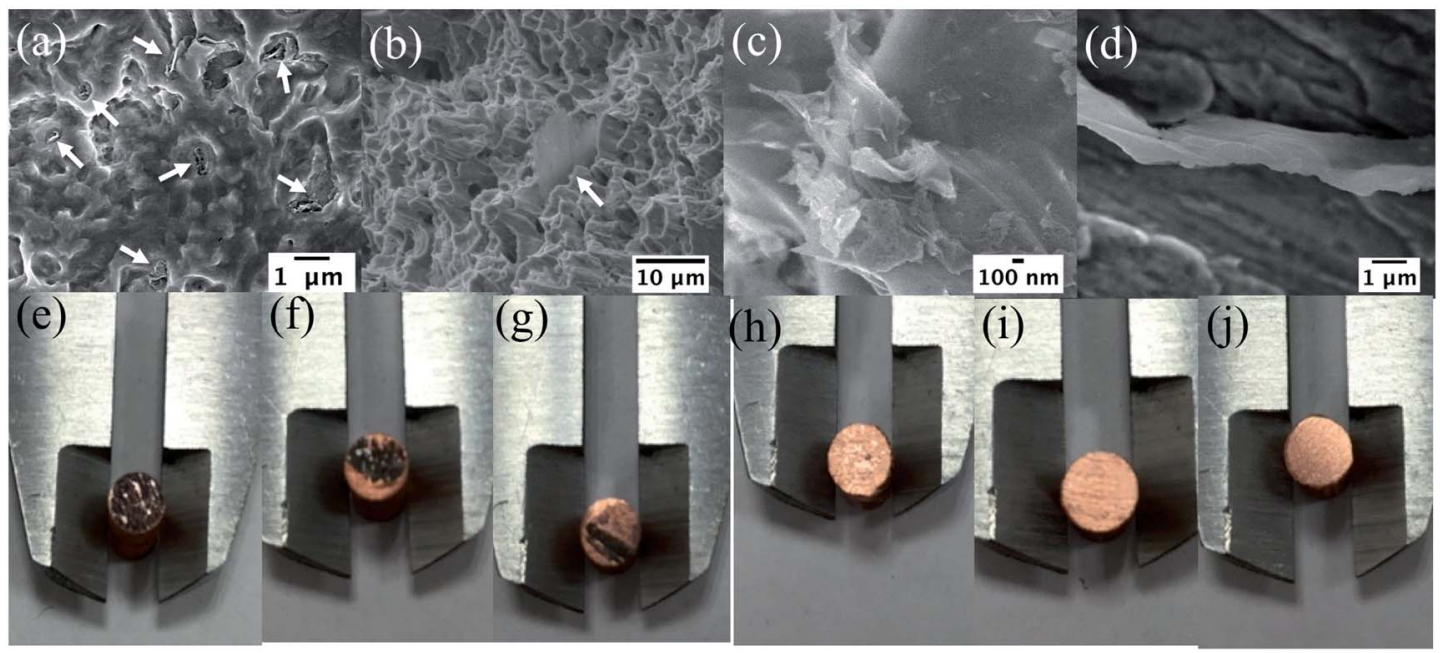

Fig. 8 SEM images of the worn ( $a$ and $d$ ) and fractured surfaces ( $b$ and $c$ ) of GPL/Cu composites and camera images of the surface after sliding. (e) Pure Cu, (f) 0.25 vol\%, (g) 0.5 vol\%, (h) 1 vol\%, (i) 1.5 vol\%, (j) 2 vol\%. 
out to be the opposite of the expected, as indicated in presented study where an addition of GPLs leads to a significant deterioration in electrical performance. ${ }^{27}$ It is suggested that the formation of a conductive network is crucial for achieving an extraordinary electrical performance. However, despite the potential formation of conductive graphitic channels at some local area, an addition of GPLs introduces porosity and interfaces within the composites, which damages the path of electron transportation and increases the possibility of electron scattering due to the decrease in the electron mean free path. ${ }^{36}$ As a result, the resistivity of composites is significantly increased with the increasing addition of the GPL content.

\section{Conclusions}

In current work, GPL/Cu composites were prepared through the SPS process and the influences of GPLs content on the tribological, thermal and electrical properties were studied. High densities, refined microstructures and uniform distribution of the GPLs are achieved for the GPL/Cu composites with varied contents of GPLs. Both tribological and thermal performances of the $\mathrm{Cu}$ matrix were significantly improved by adding the GPLs. A friction coefficient of as low as approximately 0.3 and $30 \%$ improvement in thermal conductivity were obtained by the addition of $2 \mathrm{vol} \%$ and $0.25 \%$ respectively while the electrical conductivity deteriorates regardless of content of GPLs added. The enhancement in the tribological performance is associated with the grain refinement induced by GPLs and the inherent self-lubricating property of GPLs and the thermal performance is enhanced due to a well distribution of GPLs in the Cu matrix which prevents the occurrence of GPLs agglomeration and reduces the likelihood in the formation of pores. Electrical conductivity deteriorates because of the pores formed due to GPLs breaking the continuity in the conductivity path.

\section{Conflicts of interest}

There are no conflicts to declare.

\section{Acknowledgements}

This research presented is supported by the National Natural Science Foundation of China (Grant No. 51975390, No. 51575369).

\section{References}

1 H. Bai, C. Xue, J. L. Lyu, J. Li, G. X. Chen, J. H. Yu, C. T. Lin, D. J. Lv and L. M. Xiong, Thermal conductivity and mechanical properties of flake graphite/copper composite with a boron carbide-boron nano-layer on graphite surface, Composites, Part A, 2018, 106, 42-51.

2 F. Chen, J. Ying, Y. Wang, S. Du, Z. Liu and Q. Huang, Effects of graphene content on the microstructure and properties of copper matrix composites, Carbon, 2016, 96, 836-842.

3 X. Gao, H. Yue, E. Guo, H. Zhang, X. Lin, L. Yao and B. Wang, Mechanical properties and thermal conductivity of graphene reinforced copper matrix composites, Powder Technol., 2016, 301, 601-607.

4 X. Gao, H. Yue, E. Guo, S. Zhang, L. Yao, X. Lin, B. Wang and E. Guan, Tribological properties of copper matrix composites reinforced with homogeneously dispersed graphene nanosheets, J. Mater. Process. Technol., 2018, 34, 1925-1931.

5 B. Liu, D. Zhang, X. Li, Z. He, X. Guo, Z. Liu and Q. Guo, Effect of graphite flakes particle sizes on the microstructure and properties of graphite flakes/copper composites, J. Alloys Compd., 2018, 766, 382-390.

6 J. K. Xiao, W. Zhang, L. M. Liu, L. Zhang and C. Zhang, Tribological behavior of copper-molybdenum disulfide composites, Wear, 2017, 384, 61-71.

7 Y. J. Mai, F. X. Chen, W. Q. Lian, L. Y. Zhang, C. S. Liu and $\mathrm{X}$. H. Jie, Preparation and tribological behavior of copper matrix composites reinforced with nickel nanoparticles anchored graphene nanosheets, J. Alloys Compd., 2018, 756, 1-7.

8 Y. J. Mai, M. P. Zhou, H. J. Ling, F. X. Chen, W. Q. Lian and X. H. Jie, Surfactant-free electrodeposition of reduced graphene oxide/copper composite coatings with enhanced wear resistance, Appl. Surf. Sci., 2018, 433, 232-239.

9 F. Y. Chen, J. M. Ying, Y. F. Wang, S. Y. Du, Z. P. Liu and Q. Huang, Effects of graphene content on the microstructure and properties of copper matrix composites, Carbon, 2016, 96, 836-842.

10 Z. Y. Yang, L. D. Wang, Z. D. Shi, M. Wang, Y. Cui, B. Wei, S. C. Xu, Y. P. Zhu and W. D. Fei, Preparation mechanism of hierarchical layered structure of graphene/copper composite with ultrahigh tensile strength, Carbon, 2018, 127, 329-339.

11 Y. Xiao, Z. Zhang, P. Yao, K. Fan, H. Zhou, T. Gong, L. Zhao and M. Deng, Mechanical and tribological behaviors of copper metal matrix composites for brake pads used in high-speed trains, Tribol. Int., 2018, 119, 585-592.

12 C. Ayyappadas, A. Muthuchamy, A. R. Annamalai and D. K. Agrawal, An investigation on the effect of sintering mode on various properties of copper-graphene metal matrix composite, Adv. Powder Technol., 2017, 28, 17601768.

13 K. Chu, X. H. Wang, F. Wang, Y. B. Li, D. J. Huang, H. Liu, W. L. Ma, F. X. Liu and H. Zhang, Largely enhanced thermal conductivity of graphene/copper composites with highly aligned graphene network, Carbon, 2018, 127, 102112.

14 Q. L. Che, J. J. Zhang, X. K. Chen, Y. Q. Ji, Y. W. Li, L. X. Wang, S. Z. Cao, L. Guo, Z. Wang, S. W. Wang, Z. K. Zhang and Y. G. Jiang, Spark plasma sintering of titanium-coated diamond and copper-titanium powder to enhance thermal conductivity of diamond/copper composites, Mater. Sci. Semicond. Process., 2015, 33, 67-75.

15 B. Chen, J. Yang, Q. Zhang, H. Huang, H. Li, H. Tang and C. Li, Tribological properties of copper-based composites with copper coated $\mathrm{NbSe}_{2}$ and CNT, Mater. Des., 2015, 75, 24-31.

16 P. Yang, X. You, J. Yi, D. Fang, R. Bao, T. Shen, Y. Liu, J. Tao and $\mathrm{C}$. Li, Influence of dispersion state of carbon nanotubes 
on electrical conductivity of copper matrix composites, $J$. Alloys Compd., 2018, 752, 376-380.

17 J. Liu, H. Guo, Y. Su, L. Wang, L. Wei, G. Yang, Y. Yang and K. Jiang, Spark plasma sintering of graphene platelet reinforced zirconia composites with improved mechanical performance, Mater. Sci. Eng., A, 2017, 688, 70-75.

$18 \mathrm{~J}$. Liu, H. Yan and K. Jiang, Mechanical properties of graphene platelet-reinforced alumina ceramic composites, Ceram. Int., 2013, 39, 6215-6221.

19 J. Liu, M. Wu, Y. Yang, G. Yang, H. Yan and K. Jiang, Preparation and mechanical performance of graphene platelet reinforced titanium nanocomposites for high temperature applications, J. Alloys Compd., 2018, 765, 1111-1118.

20 J. Liu, H. Yan, M. J. Reece and K. Jiang, Toughening of zirconia/alumina composites by the addition of graphene platelets, J. Eur. Ceram. Soc., 2012, 32, 4185-4193.

21 J. Liu, Y. Yang, H. Hassanin, N. Jumbu, S. Deng, Q. Zuo and K. Jiang, Graphene-Alumina Nanocomposites with Improved Mechanical Properties for Biomedical Applications, ACS Appl. Mater. Interfaces, 2016, 8, 2607-2616.

22 A. Boden, B. Boerner, P. Kusch, I. Firkowska and S. Reich, Nanoplatelet Size to Control the Alignment and Thermal Conductivity in Copper-Graphite Composites, Nano Lett., 2014, 14, 3640-3644.

23 C. Sun, X. Zhang, N. Zhao and C. He, Influence of spark plasma sintering temperature on the microstructure and strengthening mechanisms of discontinuous threedimensional graphene-like network reinforced $\mathrm{Cu}$ matrix composites, Mater. Sci. Eng., A, 2019, 756, 82-91.

24 J.-K. Xiao, L. Zhang, K.-C. Zhou and X.-P. Wang, Microscratch behavior of copper-graphite composites, Tribol. Int., 2013, 57, 38-45.

25 J. Kováčik, Š. Emmer, J. Bielek and L. u. Keleši, Effect of composition on friction coefficient of $\mathrm{Cu}$-graphite composites, Wear, 2008, 265, 417-421.

26 H. Kato, M. Takama, Y. Iwai, K. Washida and Y. Sasaki, Wear and mechanical properties of sintered copper-tin composites containing graphite or molybdenum disulfide, Wear, 2003, 255, 573-578.
27 N. Khobragade, K. Sikdar, B. Kumar, S. Bera and D. Roy, Mechanical and electrical properties of copper-graphene nanocomposite fabricated by high pressure torsion, $J$. Alloys Compd., 2019, 776, 123-132.

28 B. Duan, Y. Zhou, D. Wang and Y. Zhao, Effect of CNTs content on the microstructures and properties of $\mathrm{CNTs} / \mathrm{Cu}$ composite by microwave sintering, J. Alloys Compd., 2019, 771, 498-504.

29 K. Chu, C.-c. Jia, L.-k. Jiang and W.-s. Li, Improvement of interface and mechanical properties in carbon nanotube reinforced $\mathrm{Cu}-\mathrm{Cr}$ matrix composites, Mater. Des., 2013, 45, 407-411.

30 C. A. Isaza Merino, J. E. Ledezma Sillas, J. M. Meza and J. M. Herrera Ramirez, Metal matrix composites reinforced with carbon nanotubes by an alternative technique, $J$. Alloys Compd., 2017, 707, 257-263.

31 P. Goli, H. Ning, X. Li, C. Y. Lu, K. S. Novoselov and A. A. Balandin, Thermal Properties of Graphene-CopperGraphene Heterogeneous Films, Nano Lett., 2014, 14, 1497-1503.

32 C.-W. Nan, R. Birringer, D. R. Clarke and H. Gleiter, Effective thermal conductivity of particulate composites with interfacial thermal resistance, J. Appl. Phys., 1997, 81, 6692-6699.

33 Y. H. Qiang, S. R. Ge and Q. J. Xue, Microstructure and tribological behaviour of nitrocarburizing-quenching duplex treated steel, Tribol. Int., 1999, 32, 131-136.

34 Z. Xu, Q. Zhang, X. Huang, R. Liu, W. Zhai, K. Yang and Q. Zhu, An approximate model for the migration of solid lubricant on metal matrix self-lubricating composites, Tribol. Int., 2016, 93, 104-114.

35 J. Ordóñez-Miranda, J. J. Alvarado-Gil and R. MedinaEzquivel, Generalized Bruggeman Formula for the Effective Thermal Conductivity of Particulate Composites with an Interface Layer, Int. J. Thermophys., 2010, 31, 975-986.

36 T. Wejrzanowski, M. Grybczuk, M. Chmielewski, K. Pietrzak, K. J. Kurzydlowski and A. Strojny-Nedza, Thermal conductivity of metal-graphene composites, Mater. Des., 2016, 99, 163-173. 